\title{
The relationship of menopausal symptoms with the type of menopause and lipid levels
}

\author{
Omer Demir, Mirac Ozalp, Hidayet Sal, Turhan Aran, Mehmet A. Osmanağaoğlu \\ Department of Gynecology and Obstetrics, Karadeniz Technical University Faculty of Medicine, Trabzon, Turkey
}

\begin{abstract}
Introduction: Many postmenopausal women experience hot flashes, night sweats, decreased sexual desire and vaginal dryness. In this study, we aimed to compare the menopause symptom levels of surgical menopause patients and natural menopause patients by using a Menopause Rating Scale (MRS) and investigate whether there is a relationship between lipid levels and menopausal symptoms in surgical menopause patients.

Material and methods: This cross-sectional study was conducted on postmenopausal women who applied to the gynecology outpatient clinic. A total of 187 patients were analyzed. Of these,112 were the surgical menopause group and the remaining 75 were the natural menopause group. Total cholesterol, low-density lipoprotein cholesterol, high-density lipoprotein cholesterol and triglycerides were measured by using an enzymatic color test. In order to evaluate the postmenopausal symptoms of postmenopausal women included in the study, the MRS questionnaire adapted to the Turkish population was used.

Results: Considering the results of the MRS of the two groups, the results of surgical menopause patients were found to be statistically significantly higher. The results were statistically significantly higher in both the total score and in the two subgroups(somatic and psychological subgroups)in the surgical menopause group. When the MRS results(subgroups and total score)of women in the surgical menopause group were classified as mild and severe, no statistically significant relationship was found between symptom severity and blood lipid levels.

Conclusions: In the surgically induced menopause group, it was shown by this study that menopausal symptoms were more severe than the natural menopause group. Unlike natural menopausal patients, no relationship was found between lipid levels and severity of menopausal symptoms in surgical menopausal patients.
\end{abstract}

Key words: menopause, lipid levels, type of menopause, menopausal symptoms.

\section{Introduction}

The menopausal transition is a gradual endocrinological process in which women of reproductive age pass from the predictable menstrual periods characterized by regular, cyclic, ovulatory cycles to the last menstrual period associated with ovarian aging [1]. With the effect of hormonal changes that occur in this transition period, many postmenopausal women experience hot flashes, night sweats, insomnia, decreased sexual desire, vaginal dryness, and muscle - joint pain [2, 3]. It is known that most of these symptoms are mainly caused by estrogen deficiency $[4,5]$. However, socio-economic factors, ethnicity, and body structure have also been reported to play a role in menopausal symptoms [6-8]. There is no clear consensus on whether there is a difference between surgically induced menopause and natural menopause regarding vasomotor symptoms.

According to the results obtained from the Study of Women's Health Across the Nation (SWAN) analysis, it has been reported that serum lipid levels increase in the period between 1 year before and after the last menstrual period, which may be related to postmenopausal changes [9].

Thereupon, in a study investigating menopausal symptom levels and lipid profiles in natural menopausal patients, increased TG levels were found to be related to the level of menopausal symptoms [10].

In order to standardize menopausal symptoms, some measurement scales such as the Greene Climacteric Scale, Menopause Rating Scale (MRS), and healthrelated quality of life scale have been defined [11-13]. MRS is a scale that was developed in the 1990s with three subcategories, and a total of 11 symptoms, and was adapted to many societies over the years [14]. It was adapted to the Turkish population by Gürkan et al. [15].

In this study, we aimed to compare the menopause symptom levels of surgical menopause patients and natural menopause patients by using a MRS and investigate whether there is a relationship between lipid

Corresponding author:

Omer Demir, asst. prof., Department of Gynecology and Obstetrics, Karadeniz Technical University Faculty

Submitted: 2.04 .2020

of Medicine, Trabzon, Turkey, e-mail: itf.omerdemir@gmail.com

Accepted: 17.04 .2020 
levels and menopausal symptoms in surgical menopause patients.

\section{Material and methods}

This cross-sectional study was conducted on postmenopausal women who applied to the gynecology outpatient clinic between June 2019 and February 2020, after the approval (approval no 2019/234) of the institute ethics committee was obtained. The exclusion criteria were as follows; a history of premature menopause, thyroid disease, chemotherapy and/or pelvic radiotherapy, and hormone use. Natural menopause has been defined as the combination of the inability to have menstrual periods for twelve consecutive months without any medical reason and a follicle-stimulating hormone level above $40 \mathrm{mUI} / \mathrm{ml}$. Surgically induced menopause is defined as women who underwent bilateral oophorectomy for any reason. The number of patients included in our study between the specified dates when the inclusion criteria were met was determined to be 187 women. The main characteristics of the sample, age, gravida, parity, and the time since the last menstrual period and the time since surgery were recorded. Anthropometric measurements, weight, and height were measured while wearing light clothes without shoes. Body mass index was calculated by the ratio of kilogram $(\mathrm{kg}) /$ height $(\mathrm{m})$ square.
Venous blood samples were taken between 8:00 and 9:00 in the morning following a 12-hour fast, and plasma and serum were separated after the tubes were centrifuged at $2000 \mathrm{rpm}$ for approximately 10 minutes. Plasma and serum compartments were stored at $-80^{\circ} \mathrm{C}$ until tested. Total cholesterol, low-density lipoprotein cholesterol, high-density lipoprotein cholesterol, and triglycerides were measured by using an enzymatic color test (Beckman Coulter AU 5800 autoanalyzer).

In order to evaluate the postmenopausal symptoms of postmenopausal women included in the study, the MRS questionnaire adapted to the Turkish population was used [14] (Table 1). This form consists of 11 items, including menopausal complaints. For each item, 0: none, 1: mild, 2: moderate, 3: severe, and 4: very severe options are available. Likert type scale has three sub-dimensions. These sub-dimensions are 1 - somatic complaints subdimension (items 1 and 2), 2 - psychological complaints sub-dimension (items 3-7, 11), 3 - urogenital complaints sub-dimension (items 8-10). The minimum score that can be obtained from the scale is 0 , the highest score is 44 . The increase in the score obtained from the scale indicates the increase in the severity of the complaints. Eight for the somatic subgroup, 6 for the psychological subgroup, 3 for the urogenital subgroup, and 16 for the total score are defined as severe scores.

Data analysis was performed with SPSS version 21.0 (SPSS Inc., Chicago, IL). All data are presented as

Table 1. Menopause Rating Scale

\begin{tabular}{|c|c|c|c|c|c|}
\hline \multirow[t]{2}{*}{ Symptoms } & \multicolumn{5}{|c|}{ Score } \\
\hline & None $=0$ & Mild $=1$ & Moderate $=2$ & Severe $=3$ & Very severe $=4$ \\
\hline \multicolumn{6}{|l|}{ Hot flushes, sweating (episodes of sweating) } \\
\hline \multicolumn{6}{|l|}{$\begin{array}{l}\text { Heart discomfort (unusual awareness of heart } \\
\text { beat, heart skipping, heart racing, tightness) }\end{array}$} \\
\hline \multicolumn{6}{|l|}{$\begin{array}{l}\text { Sleep problems (difficulty in falling asleep, } \\
\text { difficulty in sleeping through, waking up early) }\end{array}$} \\
\hline \multicolumn{6}{|l|}{$\begin{array}{l}\text { Depressive mood (feeling down, sad, on the } \\
\text { verge of tears, lack of drive, mood swings) }\end{array}$} \\
\hline \multicolumn{6}{|l|}{$\begin{array}{l}\text { Irritability (feeling nervous, inner tension, } \\
\text { feeling aggressive) }\end{array}$} \\
\hline \multicolumn{6}{|l|}{ Anxiety (inner restlessness, feeling panicky) } \\
\hline \multicolumn{6}{|l|}{$\begin{array}{l}\text { Physical and mental exhaustion (general } \\
\text { decrease in performance, impaired memory, } \\
\text { decrease in concentration, forgetfulness) }\end{array}$} \\
\hline \multicolumn{6}{|l|}{$\begin{array}{l}\text { Sexual problems (change in sexual desire, } \\
\text { in sexual activity and satisfaction) }\end{array}$} \\
\hline \multicolumn{6}{|l|}{$\begin{array}{l}\text { Bladder problems (difficulty in urinating, } \\
\text { increased need to urinate, bladder } \\
\text { incontinence) }\end{array}$} \\
\hline \multicolumn{6}{|l|}{$\begin{array}{l}\text { Dryness of vagina (sensation of dryness or } \\
\text { burning in the vagina, difficulty with sexual } \\
\text { intercourse) }\end{array}$} \\
\hline $\begin{array}{l}\text { Joint and muscular discomfort (pain in the } \\
\text { joints, rheumatoid complaints) }\end{array}$ & & & & & \\
\hline
\end{tabular}


Table 2. Comparative table of demographic characteristics and blood lipid levels of groups

\begin{tabular}{lccc}
\hline Characteristic & $\begin{array}{c}\text { Natural } \\
\text { menopause } \\
(n=75) \\
\text { Mean } \pm \text { SD }\end{array}$ & $\begin{array}{c}\text { Surgically } \\
\text { induced } \\
\text { menopause } \\
(n=112) \\
\text { Mean } \pm \text { SD }\end{array}$ & $p$ \\
\hline Age & $56.7 \pm 9.3$ & $56.6 \pm 8.9$ & 0.96 \\
\hline Height $(\mathrm{m})$ & $158 \pm 4.7$ & $158 \pm 5.3$ & 0.81 \\
\hline Weight $(\mathrm{kg})$ & $79 \pm 13.9$ & $78 \pm 13$ & 0.88 \\
\hline BMD $\left(\mathrm{kg} / \mathrm{m}^{2}\right)$ & $31 \pm 5.3$ & $30 \pm 4$ & 0.98 \\
\hline Duration of & $9 \pm 8.2$ & $8.6 \pm 8.5$ & 0.72 \\
menopause (years $)$ & $143 \pm 63.2$ & $151 \pm 78.8$ & 0.48 \\
\hline TG & $57 \pm 12.6$ & $56 \pm 12$ & 0.59 \\
\hline HDL & $134 \pm 38$ & $136 \pm 41.9$ & 0.74 \\
\hline LDL & $219 \pm 44$ & $221 \pm 49.4$ & 0.75 \\
\hline Total cholesterol & & \\
\hline
\end{tabular}

SD - standard deviation, BMD - bone mineral density, TG - triglycerides, $\mathrm{HDL}$ - high-density lipoprotein, LDL - low-density lipoprotein

Table 3. Menopause Rating Scale (MRS) comparative results of natural menopause and surgical menopause groups

\begin{tabular}{lccc}
\hline Feature & $\begin{array}{c}\text { Natural } \\
\text { menopause } \\
(n=75) \\
\text { Mean } \pm \text { SD }\end{array}$ & $\begin{array}{c}\text { Surgically } \\
\text { induced } \\
\text { menopause } \\
(n=112) \\
\text { Mean } \pm \text { SD }\end{array}$ & $p$ \\
\hline Hot flushes, sweating & $1.76 \pm 1.33$ & $2.3 \pm 1.4$ & 0.02 \\
\hline Heart discomfort & $1 \pm 1.42$ & $1.5 \pm 1.7$ & 0.03 \\
\hline Somatic subscale & $2.76 \pm 2.47$ & $3.7 \pm 2.9$ & 0.01 \\
\hline Sleep problems & $1.1 \pm 1.24$ & $1.5 \pm 1.4$ & 0.03 \\
\hline Depressive mood & $1.17 \pm 1$ & $1.5 \pm 1.2$ & 0.03 \\
\hline Irritability & $1.8 \pm 1.1$ & $2.2 \pm 1.2$ & 0.03 \\
\hline Anxiety & $0.9 \pm 1.13$ & $1.4 \pm 1.3$ & 0.01 \\
\hline Physical and mental & $1.6 \pm 1.1$ & $1.9 \pm 1.2$ & 0.06 \\
exhaustion & $1.8 \pm 1.1$ & $2.1 \pm 1.2$ & 0.13 \\
\hline Joint and muscular & & & \\
discomfort & $8.5 \pm 4$ & $10.7 \pm 4.9$ & 0.001 \\
\hline Psychological subscale & $1.5 \pm 1.5$ & $1.9 \pm 1.6$ & 0.05 \\
\hline Sexual problems & $1.2 \pm 1.2$ & $1.3 \pm 1.2$ & 0.64 \\
\hline Bladder problems & $1.2 \pm 1.2$ & $1.3 \pm 1.4$ & 0.34 \\
\hline Dryness of vagina & $3.8 \pm 2.5$ & $4.5 \pm 2.8$ & 0.08 \\
\hline Urogenital subgroup & $15 \pm 5.4$ & $18.9 \pm 7.6$ & 0.00 \\
\hline Total MRS score & & &
\end{tabular}

SD - standard deviation

means and standard deviations. A one-sample Kolmogorov-Smirnov test was performed to analyze the distribution of the clinical and laboratory variables, such as age, year of menopause, BMI, and lipid profile. The student's $t$-test was used for parametric variables and the Mann-Whitney $U$ test for nonparametric variables. The outcomes were based on continuous observations of the symptom scores. For all calculations, a $p$-value of $<0.05$ was considered statistically significant.

\section{Results}

A total of 187 patients were analyzed. Of these, 112 were the surgical menopause group, and the remaining 75 were the natural menopause group. No statistically significant difference was determined between the two groups regarding age, body mass index, time since the last menstrual period/time since surgery $(p>0.05)$. No statistical difference was determined between the blood lipid levels of the two groups $(p>0.05)$. Demographic data and lipid levels of the groups are as shown in Table 2.

Considering the results of the MRS of the two groups, the results of surgical menopause patients were found to be statistically significantly higher. The results were statistically significantly higher in both the total score and in the two subgroups (somatic and psychological subgroups) in the surgical menopause group $(p<0.05)$. The MRS results of both groups are as shown in Table 3.

When the MRS results (subgroups and total score) of women in the surgical menopause group were classified as mild and severe, no statistically significant relationship was found between symptom severity and blood lipid levels $(p>0.05)$. This relationship is shown in Table 4.

\section{Discussion}

The menopausal transition period begins averagely four years before the last menstrual period and includes some physiological changes that may affect the quality of life [16-18]. In this period, when hormonal fluctuations are effective, different symptoms such as irregular menstrual cycles, hot flashes, sleep problems, mood-state fluctuations, and vaginal dryness can coexist $[17,18]$. Also, changes in blood lipid levels and the beginning of bone loss are threatening factors for longterm health. Estrogen deficiency is primarily tagged for these changes. These changes starting in the transition period, continue mostly in the postmenopausal period since the hormone level is at a minimum.

In natural menopause, the stages revealed in the STRAW study are passed in order, and the estrogen level is tried to be compensated at each stage. In surgical menopause, estrogen decreases to a minimum level since ovaries are eliminated. This situation has led us to question whether surgical menopause patients experience more severe symptoms against this non-gradual estrogen deprivation. Although there is a widespread clinical belief on this subject, no such finding was found in the only objective study investigating this issue in the literature [19]. It was shown by Aksel et al. in 1976 


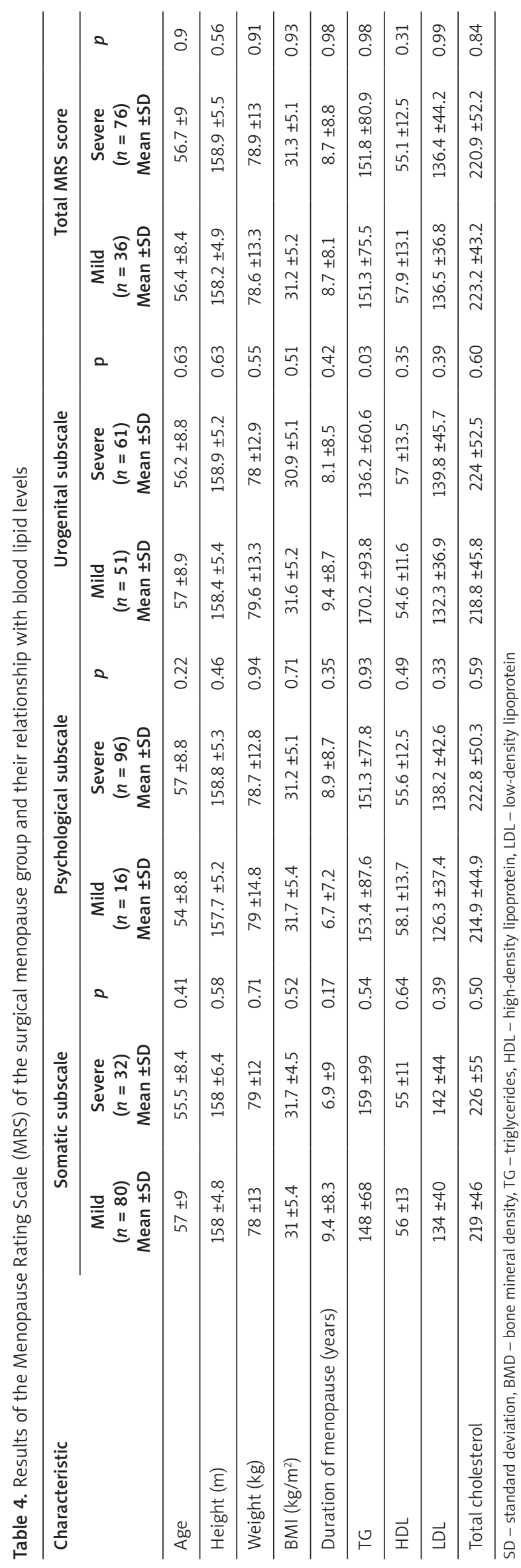

that there was no statistically significant difference regarding the hormonal changes between those who described vasomotor symptoms and those who did not [19]. However, in this study, the relationship between hormone levels observed in the early postoperative period and the presence of vasomotor symptoms was investigated. In our study, the mean time elapsed after the last menstrual period and after surgery was found to be 9 and 8.6 years, respectively, and no statistical difference was found between the compared groups. In addition, our study used a scale system and evaluated the severity of the vasomotor symptoms, not its presence/absence. This shows that our study is the first objective study to measure the severity of vasomotor symptoms comparatively in patients with surgical menopause and natural menopause.

In the current study, statistically significantly higher scores were detected in MRS somatic subgroup, psychological subgroup, and total symptom scores in surgical menopause patients compared to natural menopause patients ( $p=0.01 ; p=0.001 ; p=0.00$, respectively). No statistical difference was observed between groups only in the urogenital subgroup. The components of this subgroup include sexual problems, bladder problems, and vaginal dryness. The findings of sexual function and menopausal type studies in the literature are also supported by our study. For example, in the study published by Bıldırcın et al. in 2020 [20], it was shown that there was no significant difference regarding sexual function in patients with surgical menopause compared to patients with natural menopause. This finding corresponds to the result of our study. However, no studies were found in the literature investigating the other two components in menopause type. This study has the property of being the first study that shows no statistical significance between menopause type and bladder problems and vaginal dryness.

In previous studies, triglyceride, total cholesterol, and LDL cholesterol levels were reported to be higher in postmenopausal women compared to premenopausal women [21]. Choi et al. have shown negative changes in lipid profile since late menopausal transition period [22], Chedraui et al., on the other hand, reported that high serum TG levels lead to a decrease in sexual desire and an increase mood changes and depression rates [23]. In 2017, Kaya et al. identified statistically significantly higher TG levels in women with severe menopausal symptoms [10]. In our study, unlike the results defined in natural menopausal patients, in surgical menopause patients, any component of blood lipid levels was found to have an association with the severity of menopausal symptoms.

When the surgical menopause group was evaluated within itself, it was observed that the TG levels of the group with severe urogenital symptoms were significantly lower than the other group. It has been shown 
in the literature that the rate of atrophy is higher in the vagina, which has a low rate of fat [24]. This explains the higher urogenital symptoms.

\section{Conclusions}

As a result, in this surgically induced menopause group, it was shown by this study that menopausal symptoms were more severe than the natural menopause group. Unlike natural menopausal patients, no relationship was found between lipid levels and severity of menopausal symptoms in surgical menopausal patients. More research is needed to reveal the reasons why surgical menopause patients are more symptomatic than natural menopause patients.

\section{Disclosure}

The authors report no conflict of interest.

\section{References}

1. Harlow SD, Gass M, Hall JE, et al. Executive summary of the Stages of Reproductive Aging Workshop +10 : addressing the unfinished agenda of staging reproductive aging. Climacteric 2012; 15: 105-114.

2. Dennerstein L, Randolph J, Taffe J, et al. Hormones, mood, sexuality, and the menopausal transition. Fertil Steril 2002; 77: S42-S48.

3. Agan K, Ozmerdivenli R, Degirmenci Y, et al. Evaluation of sleep in women with menopause: results of the Pittsburg Sleep Quality Index and polysomnography. J Turk Ger Gynecol Assoc 2015; 16: 149-152.

4. Erlik Y, Meldrum DR, Judd HL. Estrogen levels of postmenopausal women with hot flashes. Obstet Gynecol 1982; 59: 403.

5. Overlie I, Finset A, Holte A. Gendered personality dispositions, hormone values, and hot flashes during after menopause. J Psychosom Obstet Gynecol 2002; 23: 219.

6. Gold EB, Bromberger J, Crawford S, et al. Factors associated with age at natural menopause in a multiethnic sample of midlife women. Am J Epidemiol 2001; 153: 865.

7. Gold EB, Colvin A, Avis N, et al. Longitudinal analysis of the association between vasomotor symptoms and race / ethnicity across the menopausal transition: study of women's health across the nation. Am J Public Health 2006; 96: 1226.

8. Augoulea A, Moros M, Kokras N, et al. Association of menopausal symptoms with sociodemographic factors and personality traits. Prz Menopauzalny 2019; 18: 191-197.

9. Derby CA, Crawford SL, Pasternak RC, et al. Lipid changes during the menopause transition in relation to age and weight: the Study of Women's Health Across the Nation. Am J Epidemiol 2009; 169: 1352-1361.

10. Kaya C, Cengiz H, Yeșil A, et al. The relation among steroid hormone levels, lipid profile and menopausal symptom severity. J Psychosom Obstet Gynaecol 2017; 38: 284-291.

11. Greene JG. Guide to the Greene Climacteric Scale. University of Glasgow, Glasgow 1991

12. Greene JG: Measuring the symptom dimension of quality oflife: General and menopause-specific scales and their sub-scale structure. In: Hormone replacement therapy and quality of life. The Parthenon Publishing Group Edited by: Schneider HPG. Boca Raton, London, New York, Washington 2002: 35-43.

13. Schneider HPG, Behre HM: Contemporary evaluation of cli-macteric complaints: Its impact on quality of life In: Hormonereplacement therapy and quality of life. The Parthenon Publishing Group, Schneider HPG (ed.). Boca Raton, London, New York, Washington 2002: 45-61.

14. Potthoff P, Heinemann LAJ, Schneider HPG, et al.. The Menopause Rating Scale (MRS II): methodological standardization in the German population. Zentralbl Gynakol 2000;122: 280-286.
15. Coban A, Nehir S, Demirci H, et al. The impact on menopausal symptoms of marital adjustment and attitude toward menopausal of married women in the climacteric period. Fırat Univ Med J Health Sci 2008; 22: 343-349.

16. Chedraui P, Perez-Lopez FR, Hidalgo L, et al. Evaluation of the presence and severity of menopausal symptoms among postmenopausal women screened for the metabolic syndrome. Gynecol Endocrinol 2014; 30: 918 924

17. Burger HG, Hale GE, Dennerstein L, Robertson DM. Cycle and hormone changes during perimenopause: the key role of ovarian function. Menopause 2008; 15: 603

18. Burger HG. Unpredictable endocrinology of the menopause transition: clinical, diagnostic and management implications. Menopause Int 2011; 17: 153

19. Aksel S, Schomberg DW, Tyrey I, Hammond CB. Vasomotor symptoms, serum estrogens and gonadotropin levels in surgical menopause. Am J Obstet Gynecol 1976; 126: 165.

20. Bıldırcın FD, Özdeş EK, Karlı P, et al. Does Type of Menopause Affect the Sex Lives of Women? Med Sci Monit 2020; 26: e921811.

21. Chang CL, Wu CH, Yao WJ, et al. Relationships of age, menopause and central obesity on cardiovascular disease risk factors in Chinese women. Int J Obes Relat Metab Disord 2000; 24: 1699-1704.

22. Choi Y, Chang Y, Kim BK, et al. Menopausal stages and serum lipid and lipoprotein abnormalities in middleaged women. Maturitas 2015; 80: 399-405.

23. Chedraui P, Hidalgo L, Chavez D, et al. Menopausal symptoms and associated risk factors among postmenopausal women screened for the metabolic syndrome. Arch Gynecol Obstet 2007; 275: 161-168.

24. Bibbo M, Camargo AC, Valeri V. Studies of Cell Lipids From the Human Vaginal and Cervical Epithelium During the Menstrual Cycle. Acta Cytol 1969; 13: 260-263. 